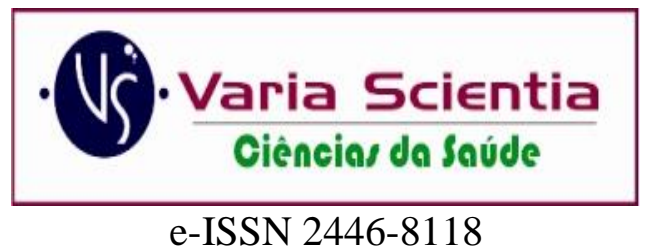

\title{
O PROFISSIONAL ENFERMEIRO NA ELABORAÇÃO DO PLANO DE PARTO NAS INSTITUIÇÕES DE SAÚDE PÚBLICA E PRIVADA
}

\section{THE NURSE PROFESSIONAL IN THE PREPARATION OF THE LABOR PLAN IN PUBLIC AND PRIVATE HEALTH INSTITUTIONS}

\section{EL PROFESIONAL DE ENFERMERÍA EN LA ELABORACIÓN DEL PLAN LABORAL EN INSTITUCIONES DE SALUD PÚBLICAS Y PRIVADAS}

\author{
Thais Emanuely Souza Santana ${ }^{1}$ \\ Vitoria Villalba Verri Wychocki ${ }^{2}$ \\ Maycon Hoffmann Cheffer ${ }^{3}$
}

\begin{abstract}
RESUMO: O plano de parto é um documento escrito, de caráter legal, onde a gestante, após informações acerca das boas práticas de atenção ao parto, pode descrever suas expectativas e desejos para vivência deste momento. Objetivo: descrever a assistência prestada no pré-natal com a elaboração do plano de parto e a visão do profissional enfermeiro e da mulher frente à construção desse instrumento. Materiais e métodos: Trata-se de um estudo exploratório, descritivo de abordagem qualitativa. Resultados: Foram identificados cinco categorias: Enfermeiro iniciando o pré-natal da rede pública; Pré-natal na rede privada centralizada na figura do médico; Plano de parto garantindo os direitos e autonomia da mulher na rede pública e privada; Plano de parto não colocado em prática no SUS pelos enfermeiros; Plano de parto colocado parcialmente em prática na rede privada pelo enfermeiro. Conclusão: O plano de parto é um instrumento conhecido entre os enfermeiros e gestantes, porém ainda há dificuldade de inserção do mesmo durante a realização do pré-natal, sendo mais adotado na rede privada que na rede pública.
\end{abstract}

DESCRITORES: Enfermagem; Pré-natal; Obstetrícia; Parto; Enfermeiras Obstetras.

\begin{abstract}
The birth plan is a written document, of a legal character, in which the pregnant woman, after information about the good practices of childbirth care, can describe her expectations and wishes for experiencing this moment. Objective: To describe the assistance provided in prenatal care with the elaboration of the delivery plan and the view of the professional nurse and the woman regarding the construction of this instrument. Materials and methods: This is an exploratory, descriptive study with a qualitative approach. Results: Five categories were identified: Nurse starting prenatal care in the public network; Prenatal care in the private network centralized in the figure of the doctor; Childbirth plan guaranteeing women's rights and autonomy in the public and private network; Delivery plan not put into practice in SUS by nurses; Birth plan partially put into practice in the private network by the nurse. Conclusion: The birth plan is a well-known instrument among

\footnotetext{
${ }^{1}$ Centro Universitário Assis Gurgacz - FAG. Enfermeira.

${ }^{2}$ Centro Universitário Assis Gurgacz - FAG. Enfermeira.

${ }^{3}$ Enfermeiro. Docente de Enfermagem do Centro Universitário Assis Gurgacz - FAG. Doutorando em enfermagem Universidade Estadual de Maringá - UEM.
} 
nurses and pregnant women; however, it is still difficult to insert it during the prenatal period, being more adopted in the private network than in the public network.

DESCRIPTORS: Nursing; Prenatal Care; Obstetrics; Parturition; Nurse Midwives.

RESUMEN: El plan de parto es un documento escrito, de carácter legal, donde la gestante, luego de recibir información sobre las buenas prácticas de atención al parto, puede describir sus expectativas y deseos para vivir este momento. Objetivo: describir la asistencia brindada en la atención prenatal con la elaboración del plan de parto y la visión de la enfermera profesional y de la mujer sobre la construcción de este instrumento. Materiais e métodos: Se trata de un estudio exploratorio, descriptivo con enfoque cualitativo. Resultados: Se identificaron cinco categorías: Enfermera iniciando atención prenatal en la red pública; Atención prenatal en la red privada centralizada en la figura del médico; Plan de parto que garantice los derechos y la autonomía de las mujeres en la red pública y privada; Plan de parto no puesto en práctica por el SUS por enfermeras; Plan de parto puesto en práctica parcialmente en la red privada por la enfermera. Conclusión: El plan de parto es un instrumento muy conocido entre enfermeras y gestantes, sin embargo, aún es difícil insertarlo durante el período prenatal, siendo más adoptado en la red privada que en la red pública.

DESCRIPTORES: Enfermería; Atención Prenatal; Obstetricia; Parto; Enfermeras Obstetrices.

\section{INTRODUÇÃO}

O parto na percepção das mulheres pode ser reconhecido como um momento único em suas vidas, em que elas passam por diversas mudanças e readaptações anatômicas, bioquímicas, fisiológicas, sociais e culturais, além de uma nova fase de mudanças e responsabilidades ${ }^{1}$.

As mulheres apresentam suas próprias concepções a respeito das vias de parto, sendo muitas vezes oriundas e relacionadas com a percepção de outras mulheres e vivências trocadas entre elas ${ }^{1}$.

O plano de parto é um documento escrito, de caráter legal, onde a gestante, após informações acerca das boas práticas de atenção ao parto, pode descrever suas expectativas e desejos para vivência deste momento, desde que este transcorra em condições normais ${ }^{2}$.

A Enfermagem tem papel crucial e desempenho ativo no que se refere ao estabelecimento de cuidados humanizados às mulheres, ajudando na fisiologia do parto, promovendo cuidado e conforto ${ }^{3}$.

A construção do plano de parto oportuniza à gestante saber melhor acerca de seus direitos e escolhas, fazendo com que ela sinta mais autonomia. Dessa forma, o enfermeiro pode auxiliar a mulher na elaboração do plano de parto, podendo aplicar mais ações educativas, auxiliando para que esta vivência aconteça de forma positiva ${ }^{2}$.

No entanto, mesmo com essa ferramenta facilitadora e importante que é o Plano de Parto, ainda há a escassez de conteúdo sobre o mesmo e também a falta de procura por conhecimento dos profissionais acerca deste assunto, e até mesmo a omissão do assunto à mulher durante o pré-natal, vendo que o procedimento ainda se encontra muitas vezes em desuso pelos enfermeiros e os demais profissionais que fazem o acompanhamento da gestante durante o pré-natal ${ }^{4}$.

A proposta desse estudo é estimular o plano de parto como uma ferramenta útil e de maior adesão pelos profissionais enfermeiros no acompanhamento do pré-natal, como uma ferramenta que auxilie a mulher e o profissional a proporcionar um atendimento mais efetivo e humanizado, seja ele em uma instituição privada ou pública. Esse estudo tem como objetivo descrever a assistência prestada no pré-natal com a construção do plano de parto na visão do profissional enfermeiro e da mulher. Frente à construção desse instrumento pressupõe-se que os profissionais enfermeiros conhecem a importância do plano de parto e realizam essa construção em conjunto com a mulher. 


\section{MATERIAIS E MÉTODOS}

Trata-se de um estudo exploratório, descritivo, e de abordagem qualitativa. A pesquisa exploratória tem como intuito disponibilizar informações sobre o assunto a ser investigado, expondo assim sua definição e delineamento, favorecendo a demarcação do tema da pesquisa, visando a definição dos objetivos e a construção das hipóteses ou revelação de um novo foco para o assunto, normalmente envolvendo um levantamento bibliográfico prévio ${ }^{5}$. Pesquisas descritivas tem em seu principal intuito a descrição das características que estão sendo buscadas como, por exemplo, a população a ser analisada, as características dessa população, o que as pessoas têm em comum e as relações que apresentam os determinados grupos ${ }^{6}$.

A pesquisa foi realizada em um município localizado na região oeste do Paraná que possui 319.608 habitantes $^{7}$. Foram participantes da pesquisa gestantes da rede pública e privada e profissionais enfermeiros da rede pública e privada. Os critérios de inclusão para as mulheres foram idade entre 18 e 37 anos e estarem acima de 35 semanas de gestação.

A coleta de dados teve início com uma doula que possuía o contato com as gestantes do grupo intitulado Gesta Cascavel, que faz parte do Gesta Paraná. A iniciativa desse grupo é fazer com que as mulheres possam trocar experiências entre elas sobre o ciclo gravídico e puerperal. Neste grupo também são realizadas palestras educativas por diversos profissionais da saúde. Atualmente o grupo conta com 60 participantes, sendo elas tanto da rede pública como da rede privada.

Em decorrência da pandemia de Covid19 o instrumento para coleta de dados foi encaminhado via WhatsApp das participantes do grupo de gestantes Gesta Cascavel que por meio do link de acesso aceitaram participar da pesquisa concordando com o Termo de Consentimento Livre e Esclarecido (TCLE), dessa maneira, o envio do formulário com perguntas semiestruturadas disponível na plataforma Google Forms pode ser preenchido. Depois de as participantes responderem o questionário, ele retornou automaticamente para a plataforma onde ficou à disposição dos pesquisadores para a análise dos dados. Foram selecionadas 28 gestantes, as quais preenchiam os critérios de inclusão.

Para os profissionais enfermeiros das redes pública e privada de saúde foi critério de inclusão ser colaborador efetivo do serviço onde trabalha. Os participantes foram orientados através de um pequeno texto enviado por Whatssap juntamente com o link que daria acesso a plataforma do Google formulários qual a primeira pergunta dispunha do TCLE deixando claro o direito de suspender a participação a qualquer momento caso se sentissem desconfortáveis ou prejudicados ao responder o questionário.

Com intuito de abranger o maior número de gestantes na rede pública os questionários também foram aplicados com encontro presencial nas unidades de saúde que se encontravam abertas devido à pandemia e a pesquisa só foi iniciada após consentimento das participantes e assinatura do TCLE.

Para a coleta de dados dos enfermeiros do SUS foi enviado o link do formulário e objetivos da pesquisa via grupo de WhatsApp, que o coordenador da atenção básica possui com todos os enfermeiros. Além disso, houve o envio via e-mail para as unidades de saúde e coleta de dados presencial nas unidades que se encontravam abertas, em ambas as tentativas o TCLE foi aplicado.

Os enfermeiros do sistema privado foram contatados via telefone e os dados foram coletados por meio dos questionários online disponibilizados via WhatsApp e e-mail após consentimento confirmado via TCLE.

Todos os questionários estiveram disponíveis para as gestantes e enfermeiros responderem entre os dias 01 de setembro e 20 de outubro de 2020. As entrevistas presenciais tiveram a assinatura do TCLE em duas vias e os questionários on-line o TCLE compôs a primeira pergunta do formulário na qual só após concordância o sistema autorizava o participante a seguir com as respostas. Os dados foram analisados por meio da estatística descritiva simples e as respostas descritas em categorias de sentido seguindo os passos: 1) Exploração do material colhido por meio do 
questionário com as participantes. 2) Agrupamento das respostas e a redação por tema. 3) Discussão dos dados obtidos com a percepção das participantes com a literatura disponível.

$\mathrm{O}$ projeto de pesquisa foi aprovado pelo Comitê de Ética em Pesquisa do Centro Universitário Assis Gurgacz de Cascavel-PR no dia 01 de setembro de 2020, com número de aprovação 4.252.837, respeitando todos os aspectos éticos de pesquisa, conforme as diretrizes da Resolução $N^{\circ} 466 / 2012$ do Conselho Nacional de Saúde.

\section{RESULTADOS}

Entre as participantes gestantes obtevese um total de 28 voluntárias, sendo elas quatro (14\%) com faixa etária de 18 a 26 anos, 24 (86\%) com faixa etária de 27 a 37 anos. Dentre elas $20(80 \%)$ recebiam atendimento na rede privada e oito (20\%) na rede pública, e nove enfermeiros, dentre eles cinco (56\%) da rede privada e quatro (44\%) da Rede Pública.

Nesta amostra, $24(86 \%)$ das gestantes conheciam o plano de parto e seus objetivos e apenas quatro (14\%) não o conheciam. Foi possível verificar que $20(71 \%)$ das gestantes não tiveram em seu pré-natal a apresentação do documento plano de parto e apenas oito (29\%) tiveram conhecimento a seu respeito.

Os dados foram classificados em cinco categorias: Enfermeiro iniciando o pré-natal da rede pública, descrevendo como o profissional enfermeiro presta o atendimento pré-natal nas redes públicas; Pré-natal na rede privada centralizada na figura do médico, nesta categoria é abordado a grande dificuldade da inserção do profissional enfermeiro nas redes privadas no acompanhamento do pré-natal; Plano de parto garantindo os direitos e autonomia da mulher na rede pública $\mathrm{e}$ privada, neste é abordado a autoconfiança, autonomia e respeito que as mulheres tem quando desenvolvem o plano de parto durante o pré-natal; Plano de parto não colocado em prática no SUS pelos enfermeiros, pode se notar ainda a falta de interesse dos profissionais da rede pública e também do sistema de saúde público em inserir essa ferramenta no dia a dia da prática profissional; Plano de parto colocado parcialmente em prática na rede privada pelo enfermeiro, nesta categoria os profissionais enfermeiros acompanham a gestante do início da gestação até o trabalho de parto e não veem necessidade em formalizar o plano de parto escrito com a gestante.

Em seus relatos as gestantes foram identificadas pela letra $\mathrm{G}$ seguida da ordem de entrevista exemplo G1, G2, G3 e os enfermeiros identificados pela letra E1, E2 e E3...

$\mathrm{Na}$ descrição das gestantes sobre a atuação do enfermeiro durante $o$ acompanhamento do pré-natal é possível perceber que ainda há pouca participação do profissional durante essa etapa. $\mathrm{Na}$ rede pública o enfermeiro tem maior contato na primeira consulta da gestante, onde ela dá entrada no pré-natal e realiza os testes rápidos e quase não participa no restante das consultas.

Categoria 1: Enfermeiro iniciando o pré-natal da rede pública.

"Tive uma consulta com enfermeira somente, a primeira consulta do pré-natal. Foi muito esclarecedora. Após isso só tive contato com o médico" $(G 1)$. "O atendimento público foi maravilhoso, as enfermeiras muito queridas e atenciosas" (G2). "Foi o profissional que iniciou meu atendimento no pré-natal, gostei bastante, me explicou todos os exames e por que tinha que fazer, atendimento excelente e compartilhou informações, foi muito atenciosa” (G3).

Na rede privada também não ocorreu o vínculo com enfermeiros, a realização do prénatal foi realizada somente com o profissional médico, conforme os relatos abaixo.

Categoria 2: Pré-natal na rede privada centralizada na figura do médico.

"Não tive acompanhamento com enfermeiro" (G1). "Não tive contato com profissional enfermeiro durante o pré-natal" (G2). "Não houve contato com enfermeiro" 
(G3). "Meu pré-natal foi realizado unicamente pelo obstetra" (G4).

Apenas duas participantes descreveram ter contato com a enfermagem pois buscaram pelo parto domiciliar e quem realizava era a enfermeira obstetra. Cabe destacar que uma gestante era da rede pública e que na insatisfação das orientações médicas buscou atendimento na rede privada.

"Excepcional, pois era enfermeira obstétrica" (G5 rede privada). "Na rede pública as questões eram incertas, porque o que a médica falava eu ia atrás e via que não era bem assim. Tudo o que eu perguntava, ela falava, a no final você saberá, tudo no final. Não gostei da profissional que me acompanhou. Ao final da gestação procurei uma enfermeira e médica particular e elas sim me esclareceram todas as dúvidas" (G6 rede pública).

É possível identificar que na descrição das gestantes o documento plano de parto é muito importante na sua construção no prénatal conforme os relatos abaixo:

Categoria 3: Plano de parto garantindo os direitos e autonomia da mulher na rede pública e privada.

"O plano de parto é um instrumento importante pois por meio dele passamos a nos informar sobre nossos direitos e com isso evitamos violência obstétrica. $\mathrm{Na}$ minha experiência, por ter um plano de parto na rede pública, sinto que fui muito mais respeitada pela equipe, pois sabiam que eu estava muito bem informada sobre tudo" (G1 rede pública). "Acho essencial o plano de parto, ele deveria sim ser construído junto com a médicalenfermeira que atende você no posto de saúde, mas não é isso o que aconteceu comigo, nem foi apresentado o plano e nem foi falado das vias de parto. Todas as informações que tive, foi pq busquei fora. O sistema Sus é completo, porém falho, bem falho, pelo menos comigo foi" (G2 rede pública).
"É importante porque para formular o plano de parto, a gestante precisa se informar $e$ tb porque no momento do parto, as informações do plano podem não ser lembradas pela gestante que está envolvida no processo" (G3 rede privada). "Sua construção foi importante, pois para isso tive q estudar e conhecer várias coisas relacionadas ao parto e principalmente ao bebê. Achei q no hospital ninguém leu aquilo. Ao entrar na sala de cirurgia (que não era o plano A, mas necessária pois já tinha contrações frequentes e bolsa rota e meu bebê continuava pélvico), expliquei $p$ a equipe de enfermagem e pediatra de plantão o q estava no plano e fui respeitada. Acho $q$ só o fato de ter um, já faz c q a equipe perceba $q V C$ está informada e sabe o q quer. Ajudou muito p mim" (G4 rede privada). "Importante para serem respeitados os seus desejos e evitar a violência obstétrica" (G5 rede privada). "Importantíssimo! Especialmente em parto hospitalar" (G6 rede privada). "É importante porque para formular o plano de parto, a gestante precisa se informar e tb porque no momento do parto, as informações do plano podem não ser lembradas pela gestante que está envolvida no processo" (G7 rede privada). "É importante para a gestante escolher a melhor via de parto com conhecimento suficiente para decidir com clareza" (G8 rede privada).

Foram nove os enfermeiros que participaram da pesquisa: quatro $(44,4 \%) \mathrm{com}$ faixa etária entre 20 e 30 anos, dois $(22,2 \%)$ entre 30 e 40 anos e três $(33,3 \%)$ entre 40 e 50 anos, sendo quatro $(44,4 \%)$ da rede pública e cinco $(55,6 \%)$ da rede privada.

Foi verificado que dois $(22 \%)$ enfermeiros da rede pública desconhecem o documento plano de parto e sete $(78 \%)$ o conhecem, sendo que apenas dois $(22 \%)$ da rede privada utilizavam essa ferramenta durante $\mathrm{o}$ atendimento de pré-natal e sete (78\%) não utilizam o documento em seus atendimentos.

Com base nas respostas é possível ver a percepção e avaliação em relação à construção do plano de parto e aplicação dele no atendimento de pré-natal na rede pública, em que foi observado que a gestante participa 
de maneira discreta dessa construção durante o pré-natal.

Categoria 4: Plano de parto não colocado em prática no SUS pelos enfermeiros.

"No meu atendimento poderia ser implementada, porém atualmente não faço" (E1). "Não tenho conhecimento a respeito do plano" (E2). "Não trabalho com plano de parto" (E3). "Não realizamos na unidade no momento" (E4).

Foi constatado que em relação à elaboração do plano de parto, ocorre a interação da gestante na realização do plano de parto com maior prevalência nas instituições privadas de saúde que o realizam.

Categoria 5: Plano de parto colocado parcialmente em prática na rede privada pelo enfermeiro

Observa-se que apenas dois enfermeiros haviam informado que realizam o plano de parto. No entanto, ao descreverem a sua atuação na prática, três enfermeiros informam que de maneira direta ou indireta acabam fazendo uso do plano de parto.

\section{"Na instituição já temos um plano de parto pré-estabelecido, seguimos os} protocolos da rede mãe paranaense e rede cegonha juntamente com o parto adequado do Albert Einstein" (E1). "Avaliamos todas as informações e a gestante define como quer que aconteça. Se há procedimentos que deseja ou não que aconteça. Isso no trabalho de parto, no parto, no pós parto imediato com ela e com seu bebê e também se for necessário a cesárea" (E2). "Vamos explicando como são e para que servem cada procedimento e quando há necessidade real de alguma coisa ser realizada. Ela tira as dúvidas e estabelece o que quer e o que não deseja que aconteça. $O$ importante é ela receber todas as informações para tomar as decisões de forma coerente $e$ consciente!" (E3). "Acho que é um instrumento valido para que a mulher gestante não sofra nenhum tipo de violência obstétrica durante o seu trabalho de parto" (E4).

\section{DISCUSSÃO}

Percebe-se que um bom percentual das gestantes, ambas da rede privada e da rede pública, conhece e busca seus direitos, podendo ser por meio de pesquisas, questionários nas consultas de pré-natal, além disso, buscam conhecer ou já conheceram o documento plano de parto.

A assistência prestada no pré-natal na elaboração do plano de parto, preconizado pela Organização Mundial de Saúde (OMS) desde 1980, é conhecido e aplicado nas unidades básicas de saúde pelos enfermeiros durante as consultas de pré-natal de baixo risco, bem como são analisados os efeitos desta repercussão durante o trabalho de parto na maternidade de referência ${ }^{8}$. Contudo, não se viu a realização destes procedimentos na prática com esse estudo

O plano de parto dá à mulher o direito de escolha e torna o momento do parto mais preparado e humanizado, podendo decidir sobre o ambiente, se querem manter ou não a ingestão de líquidos, sobre o uso ou não de analgesia, a posição que ela deseja no momento do trabalho de parto, se deseja acompanhante ou não, entre outros ${ }^{2}$. Além disso, é um instrumento ideal para ser realizado durante o pré-natal pois poderá ser construído junto da mulher e o profissional de enfermagem ou médico a fim de possibilitar que a gestante possa fazer escolhas e deixar claro suas preferências nesse momento tão marcante em sua vida ${ }^{2}$.

Apesar dos efeitos benéficos decorrentes do uso do plano de parto, notamse resistências e desafios a serem superados na utilização desta ferramenta em diferentes contextos. Em geral, o número de mulheres que o apresentam ainda é baixo. A não utilização deste instrumento pelas mulheres está relacionada, principalmente, ao desconhecimento do plano de parto e de seu propósito, além da ausência de apoio profissional necessário para entender as opções disponíveis e expressar preferências ${ }^{2}$.

Conforme Lei do Exercício Profissional de Enfermagem, o enfermeiro 
generalista possui habilidade teórico-científica para realizar assistência em pré-natal de classificação de baixo risco ${ }^{10}$. Assistência que não está presente nesse estudo por parte dos enfermeiros.

$\mathrm{O}$ enfermeiro atua no pré-natal com uma assistência focada na humanização, atendendo a gestante como um todo, levando em consideração suas crenças, valores e modos de vida, transmitindo seus saberes e criando um elo de confiança com a mulher ${ }^{11}$.

Assim, é visto que o enfermeiro tem grande importância na realização do pré-natal, e ele pode atuar de maneira geral, acompanhando a gestante em todo o período gestacional e no puerpério ${ }^{11}$. No entanto, temse visto que o profissional enfermeiro tem aparecido somente no início do pré-natal na rede pública de saúde, e em seguida as futuras mães seguem unicamente com o médico. Já na rede privada aparecem mais os enfermeiros que são especializados na área de obstetrícia, ou em diversos casos o pré-natal acontece unicamente com o médico.

Nessa direção, estudo aponta que as enfermeiras obstetras ativamente envolvidas na assistência ao parto são as profissionais ideais para apoiar a construção de um plano de parto, principalmente por terem clareza das reais possibilidades que poderão ser oferecidas às gestantes nos serviços de saúde ${ }^{12}$. Desse modo, a enfermagem precisa desenvolver ess prática e realizar uma assistência integral a saúde dessas gestantes no que diz a elaboração do plano de parto até o desfecho do parto.

O enfermeiro é capacitado e amparado legalmente para realizar ações no pré-natal e pela saúde da mulher. Com a consulta de enfermagem, é visto que a assistência prestada é imprescindível para a promoção de saúde à mulher e ao seu futuro bebê $\hat{e}^{13}$.

Embora o profissional enfermeiro seja altamente capacitado para a realização da consulta e acompanhamento no pré-natal, ainda é vista a falta de conhecimento pelas gestantes do seu real papel. $\mathrm{O}$ atendimento de enfermagem não pode ser visto como um serviço complementar ao do médico durante o pré-natal $^{13}$.

$\begin{array}{rccr}\text { Atualmente } & \text { o } & \text { pré-natal } & \text { é } \\ \text { disponibilizado à } & \text { gestante } & \text { tanto na rede }\end{array}$

pública quanto na rede privada. Entretanto, é possível notar grande diferença entre eles: o sistema público de saúde disponibiliza em média sete atendimentos à gestante durante todo o pré-natal, variando de acordo com cada caso e seu nível de classificação; já na rede privada de saúde são em média nove ou mais atendimentos, de acordo com o desejo da gestante $^{14}$.

No atendimento da rede pública as consultas acontecem com tempo mais curto, também aparecem profissionais que não realizam o acolhimento humanizado da gestante, fazendo com que ela se sinta intimidada, sendo também que os profissionais que realizam o pré-natal não serão os mesmos que estarão presentes no dia do parto. Em contrapartida, a rede privada disponibiliza um profissional à disposição a todo o tempo, para qualquer dúvida ou acontecimento do início da gestação até o parto ${ }^{15}$.

O Brasil é bem sucedido na promoção do acesso à assistência de pré-natal, a qual quase todas gestantes realizam. Mas ainda existem diversos desafios para implantar realmente um atendimento único e de qualidade. A falta de tempo e número elevado de atendimentos são dois dos fatores agravantes ${ }^{16}$. Os quais precisam ser superados.

A grande dificuldade na implementação do documento plano de parto ${ }^{2}$ na rede pública de saúde é o fato de ele ainda não ser muito reconhecido na rede, já que o método mais utilizado durante o pré-natal é a guia da rede Mãe Paranaense.

Dessa forma o enfermeiro ou médico auxilia a mulher na elaboração do plano de parto, podendo aplicar mais ações educativas, auxiliando para que esta vivência aconteça de forma positiva ${ }^{2}$.O profissional enfermeiro tem papel fundamental durante todo o período do pré-natal, pois pode acolher e acompanhar a mulher do início ao fim de sua gestação, prestando atendimento humanizado do momento do seu parto até o puerpério, assegurando o binômio mãe e filho e seu bem estar $^{17}$.

As informações trocadas entre a gestante e o enfermeiro possibilitam promover uma assistência direcionada à promoção de saúde, prevenindo doenças e agravos, e 
também ações focadas no saber e compreender o cuidado de cada uma e suas particularidades ${ }^{17}$.

Assim é possível estabelecer um vínculo de confiança, sempre com intuito de obter resultados positivos, liberdade entre ambos para esclarecimento de dúvidas e expectativas da gestante, promovendo assim uma visão da mulher em seu contexto, criando sempre condições mais positivas para 0 processo do parto $^{18}$.

\section{CONCLUSÃO}

$\mathrm{Na}$ percepção das gestantes os resultados foram positivos em relação a conhecerem seus direitos no documento plano de parto, e a buscarem sempre um atendimento melhor para elas e seus bebês, com intuito de empoderamento.

É importante ressaltar a importância que o profissional enfermeiro tem nesta área, pois pode realizar o acolhimento e acompanhar a gestante realizando a consulta de enfermagem durante toda a sua gestação, prestar um cuidado mais humanizado à gestante, assegurando seu bem-estar são ações que garantem a integralidade e continuidade do cuidado prestado.

É necessário que os profissionais enfermeiros realizem a construção do plano de parto principalmente na rede pública de saúde, proporcionando a gestante o conhecimento sobre seus direitos e escolhas para que possam atuar como protagonistas das suas histórias.

Cabe também ao profissional enfermeiro da rede pública assumir o seu papel diante do acompanhamento mensal nos acompanhamentos de pré-natal das mulheres e não somente na abertura e realização dos testes rápidos.

Durante a realização da pesquisa ficou visível a dificuldade do enfermeiro na inserção do plano de parto durante a realização das consultas de pré-natal. Um problema é a falta de conhecimento deste documento e também a falta de importância que os mesmos tem em colocar o plano de parto em prática sendo muita das vezes apenas verbalizado e não formalmente escrito. O plano de parto não é só um documento escrito ele tem um papel crucial em preparar a gestante durante toda sua gestação para esse momento único em sua vida que é momento do parto.

\section{REFERÊNCIAS}

1. Santos CL, Bortoli CFC, Prates LA, Guimarães KB, Massafera GI, Bisognin P. Preparo e percepções de gestantes sobre as vias de parto. Rev Enferm UFSM. 2016 Abr-Jun; 6 (2): 186-197.

2. Barros APZ, Lipinskin JM, Sehnem GD, Rodrigues AN, Zambiani ES. Conhecimento de enfermeiras sobre plano de parto. Rev Enferm UFSM. 2017 Jan-Fev; 7 (1): 69-79.

3. Silva TF, Costa GAB, Pereira ALF. Cuidados de enfermagem obstétrica no parto normal. Cogitare Enferm. 2011 Jan-Mar; 16 (1): 82-7.

4. Narchi NZ, Venâncio KCMP, Ferreira FM, Vieira JR. O plano individual de parto como estratégia de ensino-aprendizagem das boas práticas de atenção obstétrica. Rev esc enfermagem USP. 2019 Sep; 53:e 03518.

5. Prodanov CC, Freitas EC. Metodologia do trabalho científico: métodos e técnicas da pesquisa e do trabalho acadêmico. 2 a ed. Novo Hamburgo: Feevale; 2013.

6. Triviños ANS. Introdução à pesquisa em ciências sociais: a pesquisa qualitativa em educação. 1a ed. 22. reimpr. São Paulo: Atlas; 2013.

7. IBGE. Instituto Brasileiro de Geografia e Estatística. População no último censo. 2019. [acesso em 2020 set 19]. Disponível em: https://cidades.ibge.gov.br/brasil/pr/cascavel/ pesquisa/13/5902.

8. Santos ML, Silva DKF, Andrade PS, Albuquerque TT. Plano de Parto: O conhecimento da gestante sobre essa ferramenta o empoderamento durante a 
assistência obstétrica. Braz. J. Hea. Ver. 2020 Jul-Aug; 3 (4): 10143-10165.

9. Cortes MS, Barranco DA, Jordana MC, Roche MEM. Uso e influência dos Planos de Parto e nascimento no processo de parto humanizado. Ver Latino-am Enfermagem. 2015 Maio-Jun; 23 (3): 520-6.

10. COREN, Conselho Regional de Enfermagem. Parecer COREN-SP 034/2014. Realização da Consulta de Enfermagem para gestante de risco na Atenção Básica. 2014.

11. Botelho FS. A assistência de enfermagem ao pré-natal e sua importância. [Monografia]. Pedra Azul. Especialização em Atenção Básica em Saúde da Família]. Universidade Federal de Minas Gerais. Faculdade de Medicina. Núcleo de Educação em Saúde Coletiva. 2010.

12. Medeiros RMK, Figueiredo G, Correa ACP, Barbieri M. Repercussão do plano de parto no processo de parturição. Revista Gaúcha de enfermagem. 2019 Jun; 40: 19381447.

13. Silva CS, Souza KV, Alves VH, Cabrita BAC, Silva LR. Atuação do enfermeiro na consulta de pré-natal: limites e potencialidades. J. res.: fundam. care. 2016 Abr-Jun; 8 (2): 4087- 4098.

14. Cesar JA, Mano PS, Carlotto K, ChicaGonzalez DA, Mendoza-Sassi RA. Público versus privado: avaliando a assistência à gestação e ao parto no extremo sul do Brasil. Rev. Bras. Saude Mater. Infant. 2011 Jul-Set; 11 (3): 257-263.

15. Silva ACD, Pegoraro RF. A vivência do acompanhamento pré-natal segundo mulheres assistidas na Rede Pública de Saúde. Rev. Psicol. Saúde. 2018 Set-Dez; 10 (3): 97-107.

16. Viellas EF, Domingues RMSM, Dias MAB, Gama SGN, Filha MMT, Costa JV, et al. Assistência pré-natal no Brasil. Cadernos de Saúde Pública. 2014 Supl; 30 (1): 85-100.
17. Melo DEB, Silva SPC, Matos KKC, Martins VHS. Consulta de enfermagem no pré natal: representações sociais das Gestantes, Rev. Enferm. UFSM. 2020 Fev; 10 (18): 1-18.

18.Campos AS, Almeida ACCH, Santos RP. Crenças, mitos e tabus de gestantes acerca do parto normal. Rev Enferm UFSM. 2014 AbrMaio; 4 (2): 332-341. 